\title{
Polarized structure functions of the deuteron
}

\author{
S. Kumano * \\ Department of Physics \\ Saga University \\ Saga 840-8502, Japan
}

Talk given at the 14th International Spin Physics Symposium Osaka, Japan, October 16 - 21, 2000 (talk on Oct. 16, 2000)

* Email: kumanos@cc.saga-u.ac.jp. Information on his research is available at http://www-hs.phys.saga-u.ac.jp. 


\title{
Polarized structure functions of the deuteron
}

\author{
S. Kumano \\ Department of Physics, Saga University, Saga 840-8502, Japan
}

\begin{abstract}
Physics of spin-1 hadron is an unexplored topic in the high-energy region although spin- $1 / 2$ physics has been well investigated in the last decade. It is important to test our knowledge of hadron spin structure in a quite different field of spin physics. We discuss tensor structure functions, which do not exist for the spin- $1 / 2$ nucleon, in lepton scattering and in hadron reactions such as the polarized proton-deuteron Drell-Yan process.
\end{abstract}

\section{INTRODUCTION}

High-energy spin physics is entering into a new era in the sense that the difference between a naive quark-model prediction and experimental data is now roughly understood and that much detailed studies are in progress with the completion of the RHIC facility. So far, spin-structure studies have been focused on the spin$1 / 2$ nucleon. For higher-spin hadrons, we know that different spin physics exists such as the tensor structure in the deuteron. In order to test our understanding of the hadron structure, it is important to investigate other spin quantities. In this sense, spin-1 hadrons, for example the deuteron, should be suitable for future investigations.

In this paper, the present status is explained for the deuteron spin structure in the high-energy region. First, polarized electron-deuteron scattering is discussed briefly in connection with the deuteron spin physics. In particular, new polarized structure functions are introduced. Then, as an alternative method, the polarized proton-deuteron Drell-Yan process is discussed for finding the new distributions.

\section{SPIN-1 STRUCTURE IN ELECTRON SCATTERING}

A general formalism of the deep inelastic electron-deuteron scattering is discussed in Ref. [1]. It suggests that there exist additional structure functions in comparison with the electron-proton scattering due to the spin- 1 nature of the deuteron. We find that there are eight independent amplitudes for $\gamma\left(h_{1}\right)+d\left(H_{1}\right) \rightarrow \gamma\left(h_{2}\right)+$ $d\left(H_{2}\right)$, where $h_{1,2}$ and $H_{1,2}$ are helicities, by using momentum conservation, parity 
invariance, and time-reversal invariance. Four of them are usual structure functions: $F_{1}, F_{2}, g_{1}$, and $g_{2}$, which exist in the spin- $1 / 2$ proton. In addition, there are four new structure functions: $b_{1}, b_{2}, b_{3}$, and $b_{4}$. The $b_{3}$ and $b_{4}$ are higher-twist functions and $b_{2}$ is related to $b_{1}$ by the "Callan-Gross type" relation $b_{2}=2 x b_{1}$ in the Bjorken scaling limit, so that the most essential part of new physics is contained in $b_{1}$. A phenomenological sum rule $\int d x b_{1}(x)=0$ was proposed [2] if there is no tensor polarization for antiquark distributions; however, it does not mean that $b_{1}(x)$ itself vanishes. This sum rule is similar to the Gottfried sum rule in a way [3]. Because there is no solid reason why the antiquark tensor polarization vanishes, the violation of the above sum rule could suggest a finite tensor polarization as the Gottfriedsum-rule violation indicated a finite $\bar{u} / \bar{d}$ asymmetry.

There are three twist-2 structure functions: $F_{1}, g_{1}$, and $b_{1}$, and they are related to electron-deuteron scattering cross sections as

$$
\begin{aligned}
& F_{1} \propto\langle d \sigma\rangle \\
& g_{1} \propto d \sigma(\uparrow,+1)-d \sigma(\uparrow,-1) \\
& b_{1} \propto d \sigma(0)-[d \sigma(+1)+d \sigma(-1)] / 2 .
\end{aligned}
$$

Here, $d \sigma(\uparrow,+1)$ and $d \sigma(\uparrow,-1)$ indicate spin parallel and anti-parallel cross sections, respectively. On the other hand, $\sigma(0)$ and $d \sigma( \pm 1)$ indicate cross sections with the deuteron spin state 0 and \pm 1 . This fact means that the electron does not have to be polarized for measuring $b_{1}$. There are a number of predictions on the $x$ dependence of $b_{1}$, but we cannot discuss the model results because of the space limitation. The interested reader may find a list of papers in the reference section of Ref. [4]. At this stage, $b_{1}$ is expected to be quite small, typically $b_{1} / F_{1} \sim$ a few percent or much less. However, our experience of $g_{1}$ implies that the predictions could be wrong. In particular, the tensor structure in the high-energy region is still an unexamined topic experimentally. The HERMES experiment for measuring $b_{1}$ is in progress and its results will be reported in the near future. Therefore, the $b_{1}$ physics could become one of popular topics in hadron physics.

\section{PROTON-DEUTERON DRELL-YAN PROCESS}

In addition to the lepton scattering studies, it is, in principle, possible to investigate the same spin physics in hadron facilities. However, there are only a few theoretical studies on the spin-1 physics in hadron collisions. So far, the polarized proton-deuteron $(p d)$ Drell-Yan reaction has been investigated in connection with the tensor structure of the deuteron $[4,5]$.

Imposing the conditions of Hermiticity, parity conservation, and time-reversal invariance, we found many structure functions in the $p d$ Drell-Yan. Even after integrating the cross section over the transverse momentum of the virtual photon $\vec{Q}_{T}$ or after taking the limit $Q_{T} \rightarrow 0$, there are 22 functions [4]: 
unpolarized : $\quad W_{0,0}, W_{2,0}$

L/T polarized : $\quad V_{0,0}^{L L}, V_{0,0}^{T T}, V_{2,0}^{L L}, V_{2,0}^{T T}, U_{2,1}^{T U}, U_{2,1}^{U T}, U_{2,1}^{T L}, U_{2,1}^{L T}, U_{2,2}^{T T}$

tensor polarized : $V_{0,0}^{U Q_{0}}, V_{0,0}^{T Q_{1}}, V_{2,0}^{U Q_{0}}, V_{2,0}^{T Q_{1}}, U_{2,1}^{T Q_{0}}, U_{2,1}^{L Q_{1}}, U_{2,1}^{T Q_{2}}, U_{2,1}^{U Q_{1}}$,

$$
U_{2,2}^{U Q_{2}}, U_{2,2}^{T Q_{1}}, U_{2,2}^{L Q_{2}}
$$

The subscripts of $F_{L, M}^{\text {polp } \text { pold }_{d}}(F=W, V$, or $U)$ indicate that it is obtained by the integration $\int d \Omega Y_{L M} d \sigma /\left(d^{4} Q d \Omega\right)$. The superscripts indicate the polarization states of the proton and deuteron. The notations $U, L$, and $T$ indicate unpolarized, longitudinally polarized, and transversely polarized states. The quadrupole polarizations $Q_{0}, Q_{1}$, and $Q_{2}$ are specific in the reactions with a spin-1 hadron, and they are associated with the quadrupole terms: $Q_{0}$ for the term $3 \cos ^{2} \beta_{B}-1 \sim Y_{20}, Q_{1}$ for $\sin \beta_{B} \cos \beta_{B} \sim Y_{21}$, and $Q_{2}$ for $\sin ^{2} \beta_{B} \sim Y_{22}$ with the polarization angle of the deuteron $\beta_{B}$. The structure functions in the categories "unpolarized" and " $\mathrm{L} / \mathrm{T}$ polarized" exist in the proton-proton $(p p)$ Drell-Yan. The new functions are listed as "tensor polarized" and they are associated with the spin-1 deuteron.

In defining the spin asymmetry, we should be careful about the tensor contributions. If it is defined in the usual way, for example $A_{L L}^{\text {usual }}=[\sigma(\uparrow,-1)-\sigma(\uparrow$, $+1)] /[\sigma(\uparrow,-1)+\sigma(\uparrow,+1)]$, the denominator is not equal to the unpolarized cross section because of

$$
2<\sigma>=\sigma(\uparrow,+1)+\sigma(\uparrow,-1)+\frac{1}{3}[2 \sigma(\uparrow, 0)-\sigma(\uparrow,+1)-\sigma(\uparrow,-1)] .
$$

In order to avoid the above tensor contribution, the unpolarized cross section is used for the denominator in Ref. [4] as a "theoretical" definition of the spin asymmetry. Using the obtained $p d$ cross section with the structure functions in Eq.(2), we find the following spin asymmetries:

$$
\begin{aligned}
& <\sigma>, A_{L L}, A_{T T}, \quad A_{L T}, \quad A_{T L}, A_{U T}, A_{T U}, \\
& A_{U Q_{0}}, A_{T Q_{0}}, A_{U Q_{1}}, A_{L Q_{1}}, A_{T Q_{1}}, A_{U Q_{2}}, A_{L Q_{2}}, A_{T Q_{2}} \text {. }
\end{aligned}
$$

Those in the first line exist in the $p p$ reaction. However, the ones in the second line are specific for the $p d$ Drell-Yan, and they are related to the tensor polarizations $Q_{0}, Q_{1}$, and $Q_{2}$. For example, $A_{U Q_{0}}, A_{U Q_{1}}$, and $A_{U Q_{2}}$ are given as

$$
\begin{aligned}
A_{U Q_{0}} & =\frac{1}{2<\sigma>}\left[\sigma\left(\bullet, 0_{L}\right)-\frac{\sigma\left(\bullet,+1_{L}\right)+\sigma\left(\bullet,-1_{L}\right)}{2}\right]=\frac{2 V_{0,0}^{U Q_{0}}+\left(\frac{1}{3}-\cos ^{2} \theta\right) V_{2,0}^{U Q_{0}}}{2 W_{0,0}+\left(\frac{1}{3}-\cos ^{2} \theta\right) W_{2,0}}, \\
A_{U Q_{1}} & =\frac{\sigma\left(\bullet, I_{1}\right)-\sigma\left(\bullet, I_{3}\right)}{2<\sigma>}=\frac{\sin \theta \cos \theta \sin \phi U_{2,1}^{U Q_{1}}}{2 W_{0,0}+\left(\frac{1}{3}-\cos ^{2} \theta\right) W_{2,0}}, \\
A_{U Q_{2}} & =\frac{1}{2<\sigma>}\left[\frac{\sigma\left(\bullet, \phi_{B}=0\right)+\sigma\left(\bullet, \phi_{B}=\pi\right)}{2}-\frac{\sigma\left(\bullet, \phi_{B}=\pi / 2\right)+\sigma\left(\bullet, \phi_{B}=3 \pi / 2\right)}{2}\right] \\
& =\frac{\sin ^{2} \theta \cos 2 \phi U_{2,2}^{U Q_{2}}}{2 W_{0,0}+\left(\frac{1}{3}-\cos ^{2} \theta\right) W_{2,0}} .
\end{aligned}
$$


Here, $\theta$ and $\phi$ are polar and azimuthal angles of the $\ell^{+}$momentum. The solid circle indicates that the proton is unpolarized. The three tensor polarizations are illustrated in Fig.1.
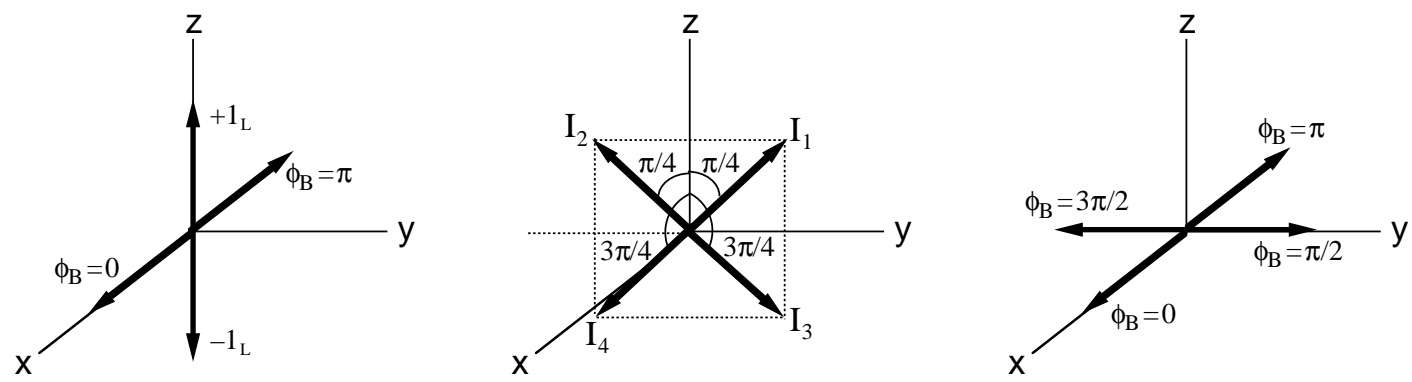

FIGURE 1. $Q_{0}, Q_{1}$, and $Q_{2}$ polarizations [4].

The $Q_{0}$ polarization asymmetry is in principle the same as the one in defining the tensor structure function $b_{1}$. On the other hand, $Q_{2}$ is associated with the tensor asymmetry in the transverse plane. The $Q_{1}$ polarization is very peculiar. The deuteron is polarized with the angle $45^{\circ}$ or $135^{\circ}$ with respect to the longitudinal axis, and the cross section difference is taken. This kind of peculiar asymmetry does not exist, of course, for the proton. This "intermediate" (according to Ref. [4]) polarization asymmetry could lead to a new unique field of spin physics. A parton-model analysis suggests that the asymmetry $Q_{0}$ should be related to $b_{1}$ type tensor distributions as [4]

$$
A_{U Q_{0}}=\frac{\sum_{a} e_{a}^{2}\left[f_{1}^{a}\left(x_{1}\right) \bar{b}_{1}^{a}\left(x_{2}\right)+\bar{f}_{1}^{a}\left(x_{1}\right) b_{1}^{a}\left(x_{2}\right)\right]}{\sum_{a} e_{a}^{2}\left[f_{1}^{a}\left(x_{1}\right) \bar{f}_{1}^{a}\left(x_{2}\right)+\bar{f}_{1}^{a}\left(x_{1}\right) f_{1}^{a}\left(x_{2}\right)\right]},
$$

where $f_{1}^{a}$ and $b_{1}^{a}$ are unpolarized and tensor-polarized distributions for the quark $a$, and $\bar{f}_{1}^{a}$ and $\bar{b}_{1}^{a}$ are those for the antiquark. The momentum fractions are given by $x_{1}$ and $x_{2}$ in the proton and the deuteron. For example, $b_{1}^{a}$ is given by $b_{1}^{a}=\left[q_{a}(0)-\right.$ $\left.\left(q_{a}(+1)+q_{a}(-1)\right) / 2\right] / 2$. We mentioned the sum rule $\int d x b_{1}(x)=0$. However, this relation crucially depends on the antiquark tensor polarization $\bar{b}_{1}^{a}$, which cannot be well probed in the electron scattering. One of the major advantages of using the $p d$ reaction is to find $\bar{b}_{1}^{a}$ rather easily, at least according to the formalism. The situation is similar to the case that the detailed $x$ dependence of $\bar{u} / \bar{d}$ was clarified by the Fermilab-E866 Drell-Yan experiment although the difference was originally indicated by the NMC data [3]. For example, in the large- $x_{F}$ region, Eq.(6) becomes

$$
A_{U Q_{0}}\left(\operatorname{large} x_{F}\right) \approx \frac{\sum_{a} e_{a}^{2} f_{1}^{a}\left(x_{1}\right) \bar{b}_{1}^{a}\left(x_{2}\right)}{\sum_{a} e_{a}^{2} f_{1}^{a}\left(x_{1}\right) \bar{f}_{1}^{a}\left(x_{2}\right)} .
$$

Therefore, the antiquark tensor distributions $\bar{b}_{1}^{a}$ can be determined if the unpolarized distributions are well known in the proton and deuteron.

Another advantage of the $p d$ Drell-Yan is that the $\bar{u} / \bar{d}$ asymmetry can be investigated for the transversity distributions [5]. Because they have chiral-odd property, 
they cannot be investigated in the inclusive electron scattering. The $p d / p p$ DrellYan ratio is given by [5]

$$
\begin{aligned}
R_{p d} \equiv \frac{\Delta_{(T)} \sigma_{p d}}{2 \Delta_{(T)} \sigma_{p p}} & =\frac{\sum_{a} e_{a}^{2}\left[\Delta_{(T)} q_{a}\left(x_{1}\right) \Delta_{(T)} \bar{q}_{a}^{d}\left(x_{2}\right)+\Delta_{(T)} \bar{q}_{a}\left(x_{1}\right) \Delta_{(T)} q_{a}^{d}\left(x_{2}\right)\right]}{2 \sum_{a} e_{a}^{2}\left[\Delta_{(T)} q_{a}\left(x_{1}\right) \Delta_{(T)} \bar{q}_{a}\left(x_{2}\right)+\Delta_{(T)} \bar{q}_{a}\left(x_{1}\right) \Delta_{(T)} q_{a}\left(x_{2}\right)\right]} \\
& \rightarrow \frac{1}{2}\left[1+\frac{\Delta_{(T)} \bar{d}\left(x_{2}\right)}{\Delta_{(T)} \bar{u}\left(x_{2}\right)}\right]_{x_{2} \rightarrow 0} \quad \text { for } x_{F} \rightarrow 1,
\end{aligned}
$$

where $\Delta_{(T)}=\Delta$ or $\Delta_{T}$ depending on the longitudinal or transverse polarization, and $\Delta_{(T)} \sigma$ indicates the polarized cross-section difference. In the second line, $x_{F} \rightarrow 1$ limit is taken together with the assumption $\Delta_{(T)} u_{v}(x \rightarrow 1) \gg$ $\Delta_{(T)} d_{v}(x \rightarrow 1)$. Leading-order numerical results are shown in Fig.2, where $r_{\bar{q}} \equiv \Delta_{(T)} \bar{u} / \Delta_{(T)} \bar{d}$. It obviously indicates that the ratio is suitable for finding the

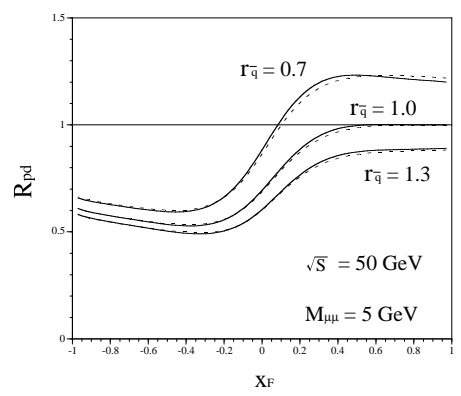
polarized light antiquark distributions, particularly in the large- $x_{F}$ region.

In this way, we found that the $p d$ Drell-Yan is an alternative way of studying the deuteron spin structure to the electron scattering. Because other studies of spin-1 physics are not discussed in this paper, the author hopes that the interested reader will look at the papers in the extensive lists of Ref. [4,5].

\section{SUMMARY}

We explained the possibilities of investigating the polarized deuteron. Exploring a new field of hadron spin, namely the tensor structure, we should be able to test our knowledge of high-energy spin physics. Because it has not been well investigated yet, we may encounter an unexpected result. Depending on the HERMES measurement in the near future, this kind of topic could become an interesting area of hadron physics. We also expressed the importance of polarized protondeuteron reactions, which are possible not only in collider experiments but also in fixed-target ones.

\section{REFERENCES}

1. P. Hoodbhoy, R. L. Jaffe, and A. Manohar, Nucl. Phys. B312, 571 (1989).

2. F. E. Close and S. Kumano, Phys. Rev. D42, 2377 (1990).

3. S. Kumano, Phys. Rep. 303, 183 (1998).

4. S. Hino and S. Kumano, Phys. Rev. D59, 094026 (1999); D60, 054018 (1999).

5. S. Kumano and M. Miyama, Phys. Lett. B497, 149 (2000). 